\title{
Determinants of isolated systolic hypertension among diabetic patients visiting the diabetic clinic at the Tamale Teaching Hospital, Northern Ghana.
}

\author{
Richard KD Ephraim, Abdul-Razak Saasi, Enoch O Anto, Prince Adoba
}

Department of Medical Laboratory Technology, School of Allied Health Sciences, College of Health and Allied sciences, University of Cape Coast, Cape Coast, Ghana

\begin{abstract}
Background: Hypertension and diabetes mellitus, two of the leading risk factors for atherosclerosis, are associated with numerous complications, including heart attacks and strokes.

Aim: This study established the prevalence and determinants of isolated systolic hypertension (ISH) in diabetes mellitus patients visiting the Tamale Teaching Hospital.

Materials and methods: In this purposive cross-sectional study, 107 diabetes mellitus patients were recruited from the out-patient diabetes clinic of the Tamale Teaching Hospital (TTH). Blood and urine samples were collected for the estimation of fasting blood glucose (FBG) and routine urinalysis respectively. A well-structured pre-tested questionnaire was used to obtain socio-demographic data and clinical history of participants, and their blood pressure measured with a mercury sphygmomanometer.

Results: The prevalence of ISH among the participants was 37.4\%. Mean age and FBG were significantly higher among participants with ISH than the normotensives $(56.20 \pm 10.60 \mathrm{v} 48.44 \pm 11.6, \mathrm{P}=0.022$; and $8.80 \pm 3.06 \mathrm{v} 6.01 \pm 0.50, \mathrm{P}=0.034 \mathrm{respec}-$ tively). Type of diabetes mellitus was associated with ISH $(\mathrm{P}=0.010)$ and age was a risk factor of ISH $(\mathrm{OR}=1.057, \mathrm{P}=0.008)$. Conclusion: Isolated systolic hypertension was prevalent in $37.4 \%$ of diabetes mellitus patients and was associated with older age. Effective measures should, therefore, be instituted to prevent ISH in patients with type 2 diabetes especially the elderly.
\end{abstract}

Keywords: Hypertension, isolated systolic hypertension (ISH), diabetes mellitus, Tamale.

DOI: http://dx.doi.org/10.4314/ahs.v16i4.33

Cite as: Ephraim RKD, Saasi A-R, Anto EO, Adoba P. Determinants of isolated systolic hypertension among diabetic patients visiting the diabetic clinic at the Tamale Teaching Hospital, Northern Ghana. Afri Health Sci.2016;16(4): 1151-1156. bttp:/ / dx.doi.org/ 10.4314/ abs.v16i4.33

\section{Introduction}

Diabetes mellitus is a clinical syndrome associated with insulin deficiency, inefficiency or both ${ }^{1}$. More than 70 per cent of people with diabetes lived in low and middle income countries, with prevalence increasing dramatically in Africa with an estimated 10.4 million people with the condition in $2007^{2}$. Diabetes mellitus is associated with an increased cardiovascular risk, and hypertension and accelerates morbidity and mortality markedly in these patients ${ }^{3}$.

Hypertension is already evident in most patients with type 2 diabetes at the time of diagnosis.

\section{Corresponding author: \\ Richard KD Ephraim, Department of Medical Laboratory Technology, University of Cape Coast +233244373839 \\ Email: rephraim@ucc.edu.gh}

Isolated systolic hypertension (ISH), is defined as systolic blood pressure (SBP) $\geq 140 \mathrm{~mm} \mathrm{Hg}$ and diastolic blood pressure (DBP) $<90 \mathrm{~mm} \mathrm{Hg}^{4}$. Central obesity is a wellknown risk factor for both diabetes diabetes mellitus and hypertension, and is thought to be a common pathway whereby obesity affects both diseases. Hypertension and diabetes substantially share common pathways such as obesity, inflammation, oxidative stress, insulin resistance, and mental stress 5 .

Isolated systolic hypertension is the most prevalent type of hypertension in adults, occurring either de novo or as a development after a long period of systolic-diastolic hypertension with or without treatment ${ }^{6}$. Several studies have demonstrated a high incidence of hypertension in type 2 diabetes mellitus or both type 1 and 2 diabetes mellitus $^{5,6,7}$. However, most of these studies looked at both diastolic and systolic hypertension among patients with diabetes.

Also, there is paucity of data on the prevalence of isolated systolic hypertension among diabetes patients (both 
type 1 and type 2) in Ghana and worldwide. This study, therefore, sought to determine the prevalence and determinants of isolated systolic hypertension among diabetes patients in the Tamale metropolis of Ghana.

\section{Materials and methods \\ Study design/ study site}

This purposive cross-sectional study was conducted from December 2011 to March 2012 at the Tamale Teaching Hospital, (TTH) in the Tamale metropolis of the Northern Region of Ghana. TTH is the largest health facility and the only teaching hospital in the northern parts of the country. It has a 400 bed capacity and provides tertiary medical care for people of the three northern regions and beyond.

\section{Participants}

A total of 107 diabetes patients who visited the diabetic clinic of the hospital within the period were recruited for the study. In order to determine the required sample size, the formula: $n=Z^{2} P Q / d 2$ was used, where, $Z=1.96$, $\mathrm{P}=$ prevalence of diabetes in Ghana i.e. 0.033 (IDF, 2014); $\mathrm{Q}=1$-P i.e. 0.967 and $\mathrm{d}=$ margin of error i.e. 0.05. Thus, the calculated sample size was $\mathrm{n}=49$. With the minimum number to be enrolled being 49, we used 107 individuals in this study. A well-structured pretested questionnaire was used to collect demographic and clinical data of patients. The authorities at the Tamale Teaching Hospital and the Institutional Review Board of University of Cape Coast (UCCIRB) approved the study. Informed written consent was sought from the participants before obtaining their data and samples.

\section{Inclusion criteria/exclusion criteria}

Type 2 diabetes patients on anti-diabetic therapy but not anti-hypertensive therapy and willing to participate were included in the study. Diabetes patients diagnosed with hypertension or those on anti-hypertensive therapy were excluded. Pregnant women, patients on other forms of therapy and patients visiting the clinic for the first time were excluded.

\section{Anthropometric measurements}

The height (to the nearest $\mathrm{cm}$ ) with height measure (Huanan measuring tape) and weight (to the nearest $\mathrm{Kg}$ ) with a weighing balance (Hospibrand ZT-120, England) were measured. The height was taken without the patient wear- ing footwear and the weight measured wearing light clothing. The Body Mass Index (BMI) was then calculated by dividing weight $(\mathrm{Kg})$ by height squared $(\mathrm{m} 2)$ and categorized according to WHO criteria into normal weight (BMI 18.5-24.9), underweight (<18.5), overweight (25.0 29.9), obese $(>30.0)$

Blood pressure measurement (using Korotkoff 1 and 5) Trained personnel using a standard cuff or a cuff appropriate with the patients size, measured blood pressure with a mercury sphygmomanometer and a stethoscope (Accoson dekamet, England), after patients had rested for at least 15 minutes. This was done in accordance with the recommendations of the American Heart Association (Kirkendall et al., 1967). Hypertension status was categorized as: optimal ( $\mathrm{SBP}<120, \mathrm{DBP}<80)$; normal $(\mathrm{SBP}=120-129, \mathrm{DBP}=80-84)$; high-normal $(\mathrm{SBP}=120$ 139 or DBP $=80-89)$; Stage 1 hypertension $(\mathrm{SBP}=140$ 159 or $\mathrm{DBP}=90-99)$; Stage 2 hypertension (SBP $>160$ or $\mathrm{DBP}>100)$; ISH $(\mathrm{SBP} \geq 140, \mathrm{DBP}<90)$ (Chobanian et al., 2003).

\section{Sample collection}

After an overnight fast (8-12 hours), $2 \mathrm{ml}$ of venous blood was collected from each participant into sodium fluoride anticoagulated tubes. The sample was centrifuged at 3000 rpm for 3 minutes and the plasma separated for the estimation of the fasting blood glucose (FBG). A freshly voided urine sample was collected from patients into a clean, dry, leak-proof and sufficiently wide necked container for urinalysis. All the biochemical parameters of routine urinalysis (urine protein, glucose, specific gravity, and $\mathrm{pH}$ ) were determined within two hours of urine collection.

\section{Estimation of plasma glucose}

The FBG level was estimated using Fortress Diagnostics reagents (Fortress diagnostics, Antrim, United Kingdom). Principle of the assay was based on the glucose oxidase/ peroxidase method (Barham and Trinder, 1972) and the colour developed was measured with a spectrophotometer (JENWAY, Bibby Scientific Ltd. Dunmow, Essex) at a wavelength of $500 \mathrm{~nm}$.

\section{Urinalysis}

The urine samples were well mixed and the urine protein, glucose, SG and $\mathrm{pH}$ were detected using a dipstick (Berotest U10, Germany). 


\section{Statistical analysis}

Data was stored in Excel and analyzed using SPSS software (version 16.0). Descriptive analysis was performed and the results expressed as means and percentages. Multiple logistic regression was done to examine the predictive factors associated with ISH. P-value $<0.05$ was considered statistically significant.

\section{Results}

Table 1 shows the demographic, clinical and biochemical data of study participants stratified by gender. Out of the 107 diabetics, 29(27.1\%) were males and 79(72.9\%) were females. Majority of both male and female participants were in their fifth decade of life, had an informal occupation, normal buminuria and type 2 diabetes. Majority of the females had ISH and were overweight.

Table 1: Socio demographic, BMI and clinical characteristics of study participants stratified by gender.

\begin{tabular}{|c|c|c|c|c|c|}
\hline Variable & Total $(\mathrm{N}=107)$ & $\begin{array}{c}\text { Male } N=29 \\
(27.1 \%) \\
\end{array}$ & $\begin{array}{c}\text { Female } N=78 \\
(72.9 \%)\end{array}$ & $X^{2}$ & $P$-value \\
\hline Age $n(\%)$ & & & & & \\
\hline$<40$ & $20(18.7)$ & $4(13.8)$ & $16(20.5)$ & 5.356 & 0.253 \\
\hline $40-49$ & $23(21.5)$ & $4(13.8)$ & $19(24.4)$ & & \\
\hline $50-59$ & $37(34.6)$ & $15(51.7)$ & $22(28.2)$ & & \\
\hline $60-69$ & $17(15.9)$ & $4(13.80$ & $13(16.7)$ & & \\
\hline$\geq 70$ & $10(9.3)$ & $2(6.9)$ & $8(10.3)$ & & \\
\hline Occupation $n(\%)$ & & & & & \\
\hline None & $5(4.7)$ & $2(6.9)$ & $3(3.8)$ & 10.372 & 0.006 \\
\hline Informal & $79(73.8)$ & $15(51.7)$ & $64(82.1)$ & & \\
\hline Formal & $23(21.5)$ & $12(41.4)$ & $11(14.1)$ & & \\
\hline BMI status $n(\%)$ & & & & & \\
\hline$<18.5$ & $5(4.7)$ & $4(13.8)$ & $1(1.3)$ & 12.400 & 0.006 \\
\hline $18.5-24.9$ & $38(35.5)$ & $14(48.3)$ & $24(30.8)$ & & \\
\hline $25-29.9$ & $35(32.7)$ & $7(24.1)$ & $28(35.9)$ & & \\
\hline$>30$ & $29(27.1)$ & $4(13.8)$ & $25(32.1)$ & & \\
\hline \multicolumn{6}{|l|}{ Albuminuria $N(\%)$} \\
\hline Normoalbuminuria & $83(77.6)$ & $22(75.9)$ & $61(78.2)$ & 0.0667 & 0.7962 \\
\hline microalbuminuria & $24(22.4)$ & $7(24.1)$ & $17(21.8)$ & & \\
\hline \multicolumn{6}{|l|}{ Type of HPT $n(\%)$} \\
\hline Optimal & $30(28.0)$ & $9(31.0)$ & $21(26.9)$ & 3.453 & 0.327 \\
\hline Normal & $18(16.8)$ & $7(24.1)$ & $11(14.1)$ & & \\
\hline High-normal & $19(17.8)$ & $6(20.7)$ & $13(16.7)$ & & \\
\hline ISH & $40(37.4)$ & $7(24.1)$ & $33(42.3)$ & & \\
\hline \multicolumn{6}{|l|}{ Type of diabetes } \\
\hline Type 1 & $10(9.3)$ & $3(10.3)$ & $7(9.0)$ & 0.047 & 0.829 \\
\hline Type 2 & $97(90.7)$ & $26(89.7)$ & $71(91.0)$ & & \\
\hline
\end{tabular}

BMI: Body Mass Index; HPT: Hypertension; ISH: Isolated systolic hypertension

Table 2 shows the mean age, clinical parameters, BMI and FBG of normotensives compared to ISH participants. Participants with ISH were older $(\mathrm{P}=0.02)$ and had FBG $(\mathrm{P}=0.034)$ than the normotensives. The mean DBP, SG and BMI were higher in participants with ISH than normotensives $(\mathrm{P}>0.05)$. The urine $\mathrm{pH}$ was, however, higher in the normotensives than those with ISH though statistically insignificant $(\mathrm{P}=0.057)$. 
Table 2: Mean age, clinical parameters, BMI and FBG of Normotensive compared to ISH participants

\begin{tabular}{lccc}
\hline Variable & Normotensive & ISH & $\boldsymbol{P}$-value \\
\hline Age & $48.44 \pm 11.6$ & $56.20 \pm 10.60$ & $\mathbf{0 . 0 2 2}$ \\
DBP & $81.10 \pm 5.83$ & $83.50 \pm 7.30$ & 0.192 \\
SG & $1.02 \pm 0.01$ & $1.030 \pm 0.04$ & 0.513 \\
Ph & $6.44 \pm 0.72$ & $6.32 \pm 0.79$ & 0.577 \\
BMI & $25.59 \pm 1.89$ & $28.80 \pm 1.71$ & 0.091 \\
FBG & $6.01 \pm 0.50$ & $8.80 \pm 3.06$ & $\mathbf{0 . 0 3 4}$ \\
\hline
\end{tabular}

Table 3 describes the association between ISH and the type of diabetes. Out of the 40 diabetics with ISH, 40
$(100 \%)$ had type II diabetes whereas none had type I diabetes. Type of diabetes was significantly associated with ISH $(\mathrm{P}=0.010)$.

Table 3: Association between ISH and the type of diabetes

\begin{tabular}{|c|c|c|c|c|}
\hline & \multicolumn{2}{|c|}{ ISH status } & \multirow[b]{2}{*}{$\mathbf{X} 2$} & \multirow[b]{2}{*}{$P$-value } \\
\hline & $\begin{array}{l}n o \\
n=67\end{array}$ & $\begin{array}{l}\text { yes } \\
\mathrm{n}=\mathbf{4 0}\end{array}$ & & \\
\hline \multicolumn{5}{|c|}{ Type of diabetes } \\
\hline Type I & $10(14.9)$ & $0(0.0)$ & 6.586 & 0.010 \\
\hline Type II & $57(85.1)$ & $40(100.0)$ & & \\
\hline
\end{tabular}

Table 4 presents the association between hypertension, their fifties and sixties and obese. Age was significantly age and BMI. Majority of the diabetics with ISH were in associated with hypertension $(\mathrm{P}=0.037)$

Table 4: Association between hypertension, Age and BMI

\begin{tabular}{|c|c|c|c|c|c|c|}
\hline \multirow[b]{2}{*}{ Variables } & \multicolumn{4}{|c|}{ Hypertension status } & \multirow[t]{2}{*}{$\left(X^{2}\right)$} & \multirow[t]{2}{*}{$P$-value } \\
\hline & $\begin{array}{l}\text { Optimal } \\
n=30\end{array}$ & $\begin{array}{l}\text { Normal } \\
n=13\end{array}$ & $\begin{array}{l}\text { High-normal } \\
n=19\end{array}$ & $\begin{array}{l}\text { ISH } \\
n=40\end{array}$ & & \\
\hline \multicolumn{7}{|l|}{ Age } \\
\hline$<40$ & $10(33.3)$ & $4(22.2)$ & $2(10.5)$ & $4(10.0)$ & 22.050 & $\mathbf{0 . 0 3 7}$ \\
\hline $40-49$ & $6(20.0)$ & $6(33.3)$ & $5(26.3)$ & $6(15.0)$ & & \\
\hline $50-59$ & $12(40.0)$ & $6(33.3)$ & $7(30.8)$ & $12(30.0)$ & & \\
\hline $60-69$ & $2(6.7)$ & $1(5.6)$ & $2(10.5)$ & $12(30.0)$ & & \\
\hline$\geq 70$ & $0(0.0)$ & $1(5.6)$ & $3(15.8)$ & $6(15.0)$ & & \\
\hline \multicolumn{7}{|c|}{ BMI status } \\
\hline$<18.5$ & $2(6.7)$ & $0(0.0)$ & $2(10.5)$ & $1(2.5)$ & 13.173 & 0.155 \\
\hline $18.5-24.9$ & $15(50.0)$ & $8(44.4)$ & $3(15.8)$ & $12(30.0)$ & & \\
\hline $25-29.9$ & $10(33.3)$ & $4(22.2)$ & $8(42.1)$ & $13(32.5)$ & & \\
\hline$>30$ & $3(10.0)$ & $6(33.3)$ & $6(31.6)$ & $14(35.0)$ & & \\
\hline
\end{tabular}


Table 5 shows the multivariate logistic regression of predictive factors of ISH among our participants. Age was a significant risk factor of $\mathrm{ISH}(\mathrm{OR}=1.057, \mathrm{P}=0.008)$. FBG, microalbuminuria, occupation and obesity were not associated with the risk of ISH ( $\mathrm{P}>0.05)$.

Table 5: Logistic regression of predictive factors associated with ISH

\begin{tabular}{lcccc}
\hline & P-value. & OR & \multicolumn{2}{c}{ 95\% C.I. } \\
Variables & & & Lower & Upper \\
\hline Age & & & 1.014 & 1.102 \\
FBG & $\mathbf{0 . 0 0 8}$ & 1.057 & 0.861 & 1.079 \\
Microalbuminuria & 0.523 & 0.964 & 0.165 & 1.286 \\
Occupation & 0.139 & 0.460 & 0.352 & 9.884 \\
Obesity & 0.273 & 3.749 & 0.186 & 1.233 \\
Constant & 0.127 & 0.478 & & \\
\hline
\end{tabular}

\section{Discussion}

Cardiovascular disease is the leading cause of morbidity and mortality among patients with diabetes mellitus (Stratton et al., 2000). In this study, we determined the prevalence and determinants of isolated systolic hypertension (ISH) in diabetics within the Tamale metropolis, Ghana. This is the first study to report the prevalence and associated risk factors of ISH among both type 1 and 2 diabetes patients in Ghana. Our findings indicate that $37.4 \%$ of type 2 diabetics had isolated systolic hypertension (ISH). FBG was significantly higher in ISH than in normotensive and age was an independent risk factor of ISH.

The prevalence of ISH among persons with type 2 diabetes observed in our study is higher than that recorded by Brenyah et al. (2013) among type 2 diabetics in a randomized cross-sectional study in Kumasi, Ghana. This could be attributed to the purposive sampling used in their study and the socio economic variations amongresidents in $\mathrm{Ku}-$ masi and Tamale. The higher literacy rate and economic status of people in Kumasi compared to Tamale might have contributed to the higher ISH prevalence found in our study compared to Brenyah et al. (2013).

Cheung and $\mathrm{Li}$ (2012) reported common pathophysiologic pathways between obesity and diabetes. This is supported by the finding of higher mean FBG among diabetespatients with ISH compared to the normotensives. In type 2 diabetes, there is insulin resistance which results in impaired lipid catabolism and obesity which can lead to the development of hypertension. Also, hyperglycaemia causes arterial stiffness and increased vascular tone resulting in systolic hypertension (Cheung and Li, 2012).

ISH was found only in type 2 diabetes. This is in consonance with Grossman \& Messerli (2008) who reported hypertension as a risk factor of type 2 diabetes. This is in agreement with the findings of Pinto (2007) who demonstrated an elevation in systolic but not diastolic pressure in patients aged 50 or over. This might be associated with structural changes in the arteries especially with large artery stiffness, and losses in arterial compliance particularly after the age of 40 (Pinto, 2007; Franklin et al., 2001). This is further supported by the logistic regression revealing age to be a risk factor for the development of ISH. The older age in type 2 diabetes therefore contributes to the high systolic blood pressure observed among them.

The co-existence of diabetes and isolated systolic hypertension in the same patient is devastating to the cardiovascular system and blood pressure control in these patients is a great challenge, since the target blood pressure is lower and the response to treatment is poor. This comorbidity results in more severe cardiomyopathy than would be expected with either hypertension or diabetes mellitus alone. The risk of macrovascular and microvascular complications in type 2 diabetes is strongly associated with systolic blood pressure (Stratton et al., 2000). Therefore, effective and easily accessible measures should be put in place to reduce the high systolic blood pressure among type 2 diabetes patients.

African Health Sciences Vol 16 Issue 4, December, 2016 
This study is limited by the use of a smaller sample size and the cross-sectional design of our study which precluded drawing of a pathophysiological causal inference of ISH in diabetes.

\section{Conclusion}

Isolated systolic hypertension was prevalent in $37.4 \%$ of diabetics and was only found in type 2 diabetes. Higher age was a risk factor of ISH. Effective measures should be adopted to prevent ISH in type 2 diabetics especially the elderly.

\section{Acknowledgement}

We acknowledge the staff of the diabetic clinic and the laboratory personnel of the Tamale Teaching Hospital for their massive support in making this work a success

\section{Conflict of interest}

The authors of this article hereby declare that there are no conflicts of interests regarding publication of this manuscript.

\section{References}

1. Stratton IM, Adler AI, Neil HAW, Matthews DR, Manley SE, Cull CA, et al.Epidemiological association of glycaemia with macrovascular and microvascular complications of type 2 diabetes (UKPDS 35). BMJ 2000; 321:405ᄀ12.

2. Ebstein M, Sowers JR: Diabetes mellitus and hypertension. Hypertension 19:403-418.1992

3. World Health Organization Guidelines Subcommittee. (1999) 1999 World Health Organization-International Society of Hypertension Guidelines for the Management of Hypertension. J Hypertens:151-83.

4. World Health Organization (2008) Theglobal burden of disease - 2004 update. Geneva, World Health Organization.

5. Barham D., Trinder P. (1972) An improved colour reagent for the determination of blood glucose by the oxidase system. Analyst. 97:142.

6. Pinto E. (2007) Blood pressure and ageing. Postgrad Med J;83:109-114.

7. Cheung B.M.Y., Li C. (2012) Diabetes and Hypertension: Is There a Common Metabolic Pathway? Curr Atheroscler Rep, 14:160-166

8. Brenyah R.C., Ephraim R.K.D., Owiredu W.K.B.A., Eghan B.A., Quaye L. (2013) Prevalence and determinants of proteinuria among type 2 diabetics in Kumasi, Ghana. Journal of Medical and Biomedical Sciences, 2(1): 13-21 Grossman E.,Franz H. Messerli F.H. (2008) Hypertension and Diabetes. Adv Cardiol., 45: 82-106

9. HarrisM.I., Zimmet P. (2007) Classification of diabetes mellitus and other categories of glucose intolerance. In: International Textbook of Diabetes mellitus, Second Edition. Ed. K.G.M.M Alberti, P. Zimmet, R.A. Detionzo and H. Keen. John Wiley and Sons Ltd. New York, pp. 9-23. http://www.idf.org/membership/afr/ghana

10. Franklin S.S., Jacobs M.J., Wong N.D., L'Italien G.J., Lapuerta P. (2001) Predominance of Isolated Systolic Hypertension Among Middle-Aged and Elderly US Hypertensives : Analysis Based on National Health and Nutrition Examination Survey (NHANES) III. Hypertension; 37(3):869-74.

11. Kirkendall W.M., Burton A.C., Epstein F.H., Freis E.D. (1967) Recommendations for human blood pressure determination by sphygmomanometers. Circulation 36(6): 980-988.

12. Bakris G.L., Sowers J.R. (2008) ASH Position Paper: Treatment of Hypertension in Patients with DiabetesAn Update. J Clin Hypertens, 10:707-713. 